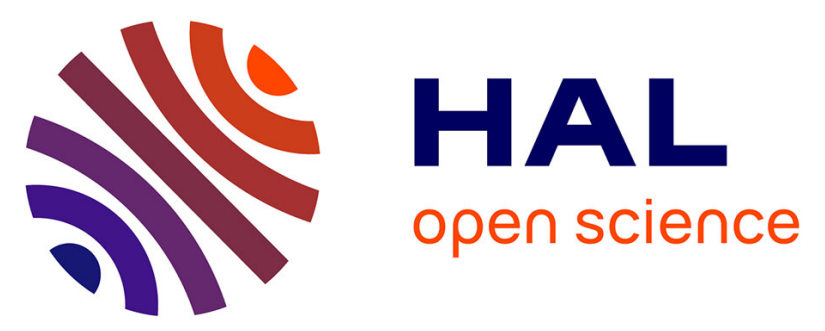

\title{
Impacto De Las Patentes Sobre El Crecimiento Económico: Un Modelo Panel Cointegrado [Impact of Patents on Economic Growth: A Cointegrated Panel Data Model] Jacobo Alberto Campo Robledo
}

\section{To cite this version:}

Jacobo Alberto Campo Robledo. Impacto De Las Patentes Sobre El Crecimiento Económico: Un Modelo Panel Cointegrado [Impact of Patents on Economic Growth: A Cointegrated Panel Data Model]. 2012. hal-00744361

\section{HAL Id: hal-00744361 \\ https://hal.science/hal-00744361}

Preprint submitted on 23 Oct 2012

HAL is a multi-disciplinary open access archive for the deposit and dissemination of scientific research documents, whether they are published or not. The documents may come from teaching and research institutions in France or abroad, or from public or private research centers.
L'archive ouverte pluridisciplinaire HAL, est destinée au dépôt et à la diffusion de documents scientifiques de niveau recherche, publiés ou non, émanant des établissements d'enseignement et de recherche français ou étrangers, des laboratoires publics ou privés. 


\section{Documentos de Trabajo}

Impacto de las Patentes sobre el crecimiento económico: Un modelo panel cointegrado

Jacobo Campo Robledo

No. 2

2012 


\title{
Impacto de las Patentes sobre el crecimiento económico: Un modelo panel cointegrado
}

\author{
Jacobo Campo Robledo ${ }^{1 *}$ \\ ${ }^{1}$ GRUPO DE ESTUDIOS ECONÓMICOS, SUPERINTENDENCIA DE INDUSTRIA Y COMERCIO, COLOMBIA.
}

\begin{abstract}
Resumen
En este artículo se presenta un modelo empírico de datos panel no estacionario y cointegrado para explicar el impacto que tiene la propiedad industrial, medida como Patentes, sobre el PIB de 10 países de América Latina en el periodo 1990 - 2010. Se aplican pruebas de raíces unitarias tradicionales y una prueba de raíz unitaria de última generación, la cual incorpora un quiebre estructural y la dependencia entre las observaciones cross-section, propuesta por Hadri y Rao (2008). A través del test de cointegración de Pedroni (1999, 2000, 2004) se prueba la existencia de una relación de largo plazo entre las variables y se estiman las elasticidades de largo plazo. Los resultados muestran la existencia de una relación positiva entre el nivel de innovación y el PIB.
\end{abstract}

Palabras claves: Crecimiento económico, Patentes, Función de Producción, Raíces Unitarias Panel, Cointegración Panel.

\begin{abstract}
This article presents an empirical model of non-stationary and cointegrated panel data to explain the impact of industrial property, measured by patents, on the GDP of 10 Latin America countries during the period 1990 to 2010. Apply traditional unit root tests and unit root test of art, which incorporates a structural break and the cross-sectional dependence, proposed by Hadri and Rao (2008). Through the Pedroni (1999, 2000, 2004) cointegration test proves the existence of a long-term relationship between variables and estimates the long-run elasticities. The results show the existence of a positive relationship between the level of innovation and GDP.
\end{abstract}

Keywords: Economic Growth, Patents, Production Function, Panel Unit Roots, Panel Cointegration.

\footnotetext{
* Magíster en Economía, miembro del Grupo de Estudios Económicos de la Superintendencia de Industria y Comercio. Cualquier error u omisión es responsabilidad exclusiva del autor y no compromete en ningún momento a la Superintendencia de Industria y Comercio. Email: jcampo@sic.gov.co
} 


\section{Introducción}

Cuando un país se encuentra en el pleno empleo de sus factores de producción, Capital y Trabajo, el residuo de Solow (1958) nos indica que los crecimientos del Producto se deben a cambios tecnológicos ya que los factores de producción no pueden incrementarse. Esta hipótesis ha sido estudiada empíricamente por numerosos investigadores a lo largo de los últimos 20 años desde la publicación de los trabajos de Barro (1991) y Mankiw, Romer y Weil (1992).

En este orden de ideas, los incrementos del PIB explicados por el residuo contienen entre otros factores, la innovación y la propiedad industrial, que a su vez tiene efectos positivos sobre el trabajo y el capital, y por ende sobre PIB.

En este documento se estudia la relación entre las Patentes y el crecimiento económico empleando un modelo de datos panel para algunos países de América Latina. A través de técnicas recientes de análisis econométrico para datos panel no estacionarios se estima la relación de largo plazo entre los factores de producción (Patentes, Capital y Trabajo) y el PIB, haciendo énfasis en el efecto que tiene el incremento de las Patentes en el crecimiento económico de los países. Lo novedoso radica en el uso de pruebas de raíces unitarias y pruebas de cointegración que controlan por heterogeneidad, dependencia cross-country y quiebre estructural, como la pruebas de Hadri y Rao (2008). En concreto, con el fin de determinar el orden de integración de las series se aplican pruebas de raíces unitarias de primera generación, como la IPS (2003), LLC (2002), Maddala y Wu (1999) (Fisher tipo ADF) y Choi (2001) (Fisher tipo PP), Breitung (2000), Hadri (2000), y de segunda generación, como la prueba propuesta por Hadri y Rao (2008), las cuales a diferencia de las de primera controlan por dependencia cross-country y quiebre estructural. Posteriormente, se aplica la prueba de cointegración de Pedroni, con el fin de determinar la existencia o no de una relación de largo plazo entre las variables por medio del estimador de FMOLS, y poder estimar los coeficientes para cada país.

Para propósitos de Política, es importante conocer si existe una relación de largo plazo entre el PIB y el número de Patentes, con el fin de conocer la causalidad que existe entre estas dos series, la cual se supone es bidireccional, es decir, existe cierto "Feedback" entre el incremento de las Patentes y el crecimiento económico.

Este documento está organizado de la siguiente manera. En la sección 2 se presenta una síntesis de estudios empíricos que se han llevado a cabo a nivel internacional sobre la relación entre patentes y crecimiento económico. La sección 3 presenta el modelo y la metodología para estimar. Los resultados de las pruebas de raíces unitarias y de Cointegración se presentan y analizan en la sección 4. Finalmente, se concluye en la quinta y última sección. 


\section{Relación Patentes y Crecimiento Económico}

La relación entre las patentes y el crecimiento económico de las economías ha sido un tema bastante estudiado durante las últimas dos décadas, aproximadamente. Esta sección busca presentar estudios a nivel internacional que se han enfocado en esta relación, es decir, el nexo existente entre patentes y crecimiento económicos de los países. La literatura existente permite agrupar estos estudios en 2 clases, los que determinan un efecto directo que va de las patentes al crecimiento económico, y los que determinan un efecto indirecto entre estos. Indirecto, cuando afectan el crecimiento económico a través de otro factor de producción, como el capital o el trabajo, por ejemplo Gould y Gruben (1996) a través de un modelo de corte transversal sobre protección de patentes estudian el rol que juegan los derechos de propiedad intelectual sobre el crecimiento económico de un país. Sus resultados presentan evidencia empírica que van en la vía de asegurar que la propiedad intelectual es un determinante del crecimiento económico, y el efecto de las patentes es mayor en países más abiertos, en términos relativos. Adicionalmente, Park y Ginarte (1997) demuestran que las patentes tienen un impacto positivo sobre la acumulación de capital, y por ende, al incrementar el capital fijo tiene un efecto positivo sobre el crecimiento económico de las economías.

Koléda (2004) muestra que el efecto de los requerimientos de patente novedosa sobre el crecimiento del PIB puede exhibir una forma de U invertida, implicando que una política de protección de propiedad intelectual más fuerte puede frenar el crecimiento económico de una economía, y demostrando que existe un nivel óptimo de requerimientos el cual maximiza el crecimiento económico.

Según el estudio de Fink y Maskus (2005), la posibilidad de que el efecto de los derechos de propiedad intelectual sobre el crecimiento económico de los países, dependa del nivel de desarrollo económico es alta. A este hallazgo se suman los resultados de Schneider (2005), obtenidos a través de un modelo de datos panel de 47 países desarrollados y en desarrollo entre 1970 y 1990, los cuales sostienen que unos derechos de patentes más fuertes legalmente tienen un efecto positivo sobre la innovación, y por tanto sobre el crecimiento económico, solamente en países desarrollados. Por otro lado, Chen y Puttitanun (2005) empleando datos panel de 64 países en desarrollo obtienen resultados a favor de que unos derechos de patentes más fuertes tienen un efecto positivo sobre la innovación en economías en desarrollo. Adicionalmente, presentan evidencia empírica sobre la existencia de una relación en forma de U entre los derechos de propiedad intelectual y el crecimiento económico, primero decrece y después aumenta.

Futagami y Iwaisako (2007) demuestran que un modelo con patentes de duración infinita no maximiza el bienestar social, mientras que un modelo endógeno de duración finita, que no presenta efectos de escala en la función de producción, maximiza el bienestar social ${ }^{1}$. Cysne y Turchick (2012) estudian el nivel óptimo de protección de derechos de propiedad intelectual a

\footnotetext{
1 Autores como Tandon (1982), Judd (1985), Gilbert y Shapiro (1990) han estudiado este tipo de modelos de crecimiento con una política de duración de patentes infinita.
} 
través de un modelo de crecimiento de investigación y desarrollo $(\mathrm{I}+\mathrm{D})$ con una tasa exógena de imitación.

Adicionalmente, Hu y Png (2012) llevan a cabo un análisis empírico sobre el efecto que tienen los derechos de patentes sobre la innovación y el crecimiento económico. A través de la metodología de diferencias en diferencias estudian el impacto que tienen los cambios en materia de derechos de patentes, con un modelo de datos panel de 54 industrias manufactureras de 72 países durante el periodo 1981 - 2000. Sus resultados indican que las industrias que son intensivas en patentes responden de forma más fuerte (crecimiento mayor) a las leyes de patentes que las otras industrias.

En un reciente estudio, Kim et al. (2012) por medio de dos modelos estudian el efecto de las patentes y los modelos de utilidad sobre la innovación y el crecimiento económico, controlando a su vez por nivel de desarrollo económico (tecnológico). Primero estudian un modelo a nivel de países, por medio de un modelo de datos panel, para el periodo 1975 - 2003, y después un modelo a nivel de empresas en Korea, para el periodo 1970 - 1995 empleando 13.530 firmas. Sus conclusiones aseguran que la protección de patentes contribuye a la innovación y al crecimiento económico de los países desarrollados, sin embargo no ocurre igual con los países en desarrollo ya que no encuentran evidencia estadística. Lo anterior es un resultado del estudio de Kim (1997), el cual sostiene que la protección de patentes es importante para la actividad industrial cuando las economías han alcanzado cierto nivel de su capacidad innovadora, acompañado de una extensa infraestructura en ciencia y tecnología. Paralelamente, a nivel de empresas, las innovaciones que son patentables evidencian un efecto positivo y significativo sobre el crecimiento de la empresa.

Finalmente, Campo et al. (2012) presentan la importancia de la presencia de oficinas de propiedad industrial en las regiones de Colombia, ya que estas pueden mejorar el uso del Sistema de Propiedad Industrial y contribuir al desarrollo de las regiones. A través de la metodología de componentes principales, presentan un índice de regionalización (IROPI) con el fin de ubicar estratégicamente 6 oficinas de propiedad industrial a lo largo del territorio nacional y dinamizar el desarrollo regional.

\section{Metodología y Modelo Econométrico}

El presente documento, como se ha mencionado, tiene como objetivo estimar la relación que existe entre la propiedad industrial, medida como número de patentes, y el PIB real para 10 países de América Latina, durante el periodo comprendido entre 1990 - 2010. Durante las últimas dos décadas, los datos panel han sido utilizados como herramienta de análisis por parte de investigadores de diversas áreas para estudiar las relaciones entre diferentes variables. La razón principal es que esta metodología combina una dimensión de tiempo (series de tiempo) con otra transversal (Corte Transversal), lo cual tiene mayores bondades al momento de hacer inferencia estadística. 
En este orden de ideas, al trabajar con datos panel macroeconómico, en los cuales la serie de tiempo es mayor a la cantidad de individuos, se debe tener en cuenta la existencia de una relación de largo plazo entre las variables que se analizan para el grupo de individuos. En otras, palabras, debemos asegurar que exista una relación de cointegración para evitar el problema de obtener resultados espurios como lo sostienen Engle y Granger (1987). Entarf (1997), Kao (1998) y Phillips y Moon (1999), introdujeron el término de relaciones espurias en el uso de datos panel, cuando las observaciones de tiempo son mayores al número de individuos en un panel.

El modelo a estimar entonces, es el siguiente

$$
\operatorname{Ln}(Y)_{i t}=\alpha_{i}+\beta_{1} \operatorname{Ln}(K)_{i t}+\beta_{2} \operatorname{Ln}(L)_{i t}+\beta_{3} \operatorname{Ln}(P a t)_{i t}+\varepsilon_{i t}
$$

Donde $\operatorname{Ln}\left(Y_{i t}\right)$ es el logaritmo del PIB de cada país $(i)$ en el periodo $(t), \operatorname{Ln}\left(K_{i t}\right)$ es el logaritmo de la formación bruta de capital fijo de cada país $(i)$ en el periodo $(t), \operatorname{Ln}\left(L_{i t}\right)$ es el logaritmo de la fuerza laboral de cada país $(i)$ en el periodo $(t)$, y $\operatorname{Ln}\left(\right.$ Pat $\left._{i t}\right)$ es el logaritmo de los registros de Patentes de cada país $(i)$ en el periodo $(t)$.

\subsection{Pruebas de Raíces Unitarias Tradicionales}

Se considera inicialmente el orden de integración de las series PIB, Capital, Trabajo y Patentes, expresadas en logaritmos. Inicialmente, se emplean las pruebas para datos panel conocidas como de Primera Generación, como las desarrolladas por Im et al. (2003), Levin et al. (2002), Breitung (2000), Maddala y Wu (1999) (Fisher tipo ADF) y Choi (2001) (Fisher tipo PP). Las pruebas de Maddala y Wu (1999) e IPS (2003) permiten la heterogeneidad entre los individuos de los datos del panel. Estas pruebas de raíces unitarias para panel tienen su fundamento en las pruebas desarrolladas para series de tiempo, pero tienen una ventaja sobre estas últimas, y es que al combinar series de tiempo y datos de corte transversal se obtienen más grados de libertad lo cual mejora las propiedades de los estimadores, y además corrigen la heterogeneidad no observada.

\subsection{Prueba de Raíces Unitarias de Hadri y Rao (2008)}

Se emplea, además de las pruebas tradicionales, una prueba de raíz unitaria de segunda generación, la cual tiene en cuenta la dependencia cruzada entre los individuos del panel (en específico, entre los países). De igual forma, esta prueba incorpora la presencia de un quiebre estructural en la serie. Cabe resaltar, que algunos trabajos empíricos sobre el tema no tienen en cuenta que este tipo de análisis está muy influenciado por el supuesto de estabilidad en los parámetros del modelo. Es bien conocido que la mayoría de las series macroeconómicas presentan cambios estructurales, en nivel, pendiente o en ambas, y en este caso el uso de pruebas de raíces unitarias están sesgadas a no rechazar la hipótesis nula de no estacionariedad (raíz unitaria). En este orden de ideas, se 
aplica la prueba desarrollada por Hadri y Rao (2008) quienes extienden la prueba de Hadri (2000) ${ }^{2}$ e incorporan la presencia de cambio estructural y la dependencia cruzada existente entre los individuos del panel.

Hadri y Rao (2008) establecen los siguientes 4 tipos de modelos, basándose en los modelos propuestos por Perron (1989):

$$
\begin{gathered}
\text { Modelo 0: } y_{i t}=\alpha_{i}+r_{i t}+\delta_{i} D_{i t}+\varepsilon_{i t} \\
\text { Modelo 1: } y_{i t}=\alpha_{i}+r_{i t}+\delta_{i} D_{i t}+\beta_{i t} t+\varepsilon_{i t} \\
\text { Modelo 2: } y_{i t}=\alpha_{i}+r_{i t}+\gamma_{i} \delta_{i} D T_{i t}+\beta_{i t} t+\varepsilon_{i t} \\
\text { Modelo 3: } y_{i t}=\alpha_{i}+r_{i t}+\delta_{i} D_{i t}+\gamma_{i} \delta_{i} D T_{i t}+\beta_{i t} t+\varepsilon_{i t}
\end{gathered}
$$

donde $\left(y_{i t}\right)$ son las series a las cuales se les aplica el test, $i=1, \ldots, N$ y $t=1, \ldots, T$. Adicionalmente, $\left(r_{i t}\right)$ corresponde a un camino aleatorio sin intercepto $\left(r_{i t}=r_{i t-1}+v_{i t}\right)$. Los coeficientes $\left(\alpha_{i}\right),\left(\beta_{i}\right),\left(\delta_{i}\right)$ y $\left(\gamma_{i}\right)$ son desconocidos. Los errores $\left(v_{i t}\right)$ y $\left(\varepsilon_{i t}\right)$ son i.i.d. $\mathrm{y}$ mutuamente independientes $\mathrm{y},\left(D_{i t}\right)$ y $\left(D T_{i t}\right)$ las variables dummy

$$
\begin{gathered}
D_{i t}=\left\{\begin{array}{lll}
1 & \text { si } & t>T_{B, i} \\
0 & \text { o.w. }
\end{array}\right. \\
D T_{i t}=\left\{\begin{array}{ccc}
t-T_{B, i} & \text { si } & t>T_{B, i} \\
0 & & \text { o.w. }
\end{array}\right.
\end{gathered}
$$

Donde $T_{B, i}=\omega_{i} T$, con $\omega_{i} \in(0,1)$, indica el lugar del tiempo en que ocurre el quiebre para cada individuo del panel. El estadístico Multiplicador de Lagrange (LM) es mejorado para permitir la presencia de un quiebre estructural en el panel de datos, y se define como:

$$
\widehat{L M}_{T, N, k}(\hat{\omega})=\frac{1}{N} \sum_{i=1}^{N} \eta_{i, T, k}\left(\hat{\omega}_{i}\right)
$$

donde $\left(\omega_{i}\right)$ denota que el estadístico se ha construido para un valor específico del quiebre para cada individuo. El parámetro $\eta_{i, T, k}\left(\hat{\omega}_{i}\right)$ es el estadístico para cada serie de tiempo propuesto por Hadri (2000) y permite un quiebre estructural bajo la hipótesis nula. El subíndice $(k)$ denota los 4 modelos considerados por Hadri y Rao (2008) $(\mathrm{k}=0,1,2,3)$. $S_{i t}^{2}$ es la suma parcial de los errores, y

\footnotetext{
2 Esta prueba de estacionariedad se basa en el estimado LM propuesto por KPSS (1992) en la prueba de estacionariedad para series de tiempo.
} 
$\sigma_{\varepsilon, i}^{2}$ es un estimador de la varianza de largo plazo (LRV) de los errores $\left(\varepsilon_{i t}\right)$ de la ecuación apropiada ((2),(3),(4),(5))

$$
\hat{\sigma}_{\varepsilon, i}^{2}=\lim _{T \rightarrow \infty} T^{-1} E\left(S_{i t}^{2}\right)
$$

El estadístico de la ecuación (6) sigue una distribución normal

$$
Z_{k} \hat{\omega}_{i}=\frac{\sqrt{N}\left(\widehat{L M}_{T, N, k}\left(\hat{\omega}_{i}-\bar{\xi}_{k}\right)\right)}{\bar{\zeta}_{k}}
$$

Donde $\quad \bar{\xi}_{k}=\frac{1}{N} \sum_{i=1}^{N} \xi_{i, k} \quad$ y $\quad \bar{\zeta}_{k}=\frac{1}{N} \sum_{i=1}^{N} \zeta_{i, k}^{2}$ son la media y la varianza del estimador, respectivamente. Para corregir la presencia de una posible correlación serial, Hadri y Rao (2008) proponen seguir la metodología de Sul et al. (2005), la cual consiste básicamente en especificar un modelo $A R(p)$ para los errores estimados $\left(\hat{\varepsilon}_{i t}\right)$ del modelo con quiebre que se haya seleccionado, es decir:

$$
\hat{\varepsilon}_{i t}=\rho_{i, 1} \hat{\varepsilon}_{i t-1}+\rho_{i, 2} \hat{\varepsilon}_{i t-2}+\rho_{i, 3} \hat{\varepsilon}_{i t-3}+\cdots+\rho_{i, p_{i}} \hat{\varepsilon}_{i t-p_{i}}+v_{i t}
$$

La varianza de largo plazo (LRV) se estima para obtener el estimado de esta $\left(\hat{\sigma}_{\varepsilon_{i}}^{2}\right)$ con la siguiente condición

$$
\hat{\sigma}_{\varepsilon_{i}}^{2}=\min \left\{T \hat{\sigma}_{v_{i}}^{2}, \frac{\hat{\sigma}_{v_{i}}^{2}}{\left(1-\hat{\rho}_{i}(1)\right)^{2}}\right\}
$$

Donde $\hat{\rho}_{i}(1)$ es la suma de todos los coeficientes autorregresivos estimados en la ecuación $7, \hat{\sigma}_{v_{i}}^{2}$ como se mencionó anteriormente, es la varianza de largo plazo (LRV) estimada de los errores $\left(\hat{\varepsilon}_{i t}\right)$ de la misma ecuación. El número de rezagos óptimos se determina empleando el criterio de selección Bayesiano Schwarz (SBIC).

De igual manera, para corregir la existencia de una dependencia cruzada entre los individuos, proponen seguir una metodología bootstrap que es presentada por Maddala y Wu (1999), y estimar los errores $\left(\hat{v}_{i t}^{*}\right)$, para obtener $\left(\hat{v}_{i t}\right)$ a través del bootstrap. El error $\left(\hat{\varepsilon}_{i t}^{*}\right)$ se genera de manera recursiva, a saber:

$$
\hat{\varepsilon}_{i t}^{*}=\rho_{i, 1} \hat{\varepsilon}_{i t-1}^{*}+\rho_{i, 2} \hat{\varepsilon}_{i t-2}^{*}+\rho_{i, 3} \hat{\varepsilon}_{i t-3}^{*}+\cdots+\rho_{i, p_{i}} \hat{\varepsilon}_{i t-p_{i}}^{*}+v_{i t}^{*}
$$


Finalmente, siguiendo a Chang (2004) se obtiene $\left(y_{i t}^{*}\right)$ introduciendo $\left(\hat{\varepsilon}_{i t}^{*}\right)$ en el componente determinístico del modelo correspondiente que se haya seleccionado inicialmente ((2), (3), (4); (5)), para derivar la distribución empírica del estimador LM, el procedimiento bootstrap se repite 1000 veces.

\subsection{Prueba de Cointegración}

Luego de comprobar que las series son integradas de orden uno, es decir, que contienen una raíz unitaria en el panel, continuamos con la prueba de cointegración, con el fin de encontrar evidencia sobre la existencia de una relación entre las variables en el largo plazo. Lo anterior se prueba empleando la conocida prueba de cointegración panel heterogéneo de Pedroni (1999, 2000, 2004). Esta prueba se basa en 2 grupos conformados por 7 estadísticos, el primero de estos está basado en el estadístico de Phillips y Ouliaris (1990), definido como sigue

$$
\tilde{Z}_{p}=\sum_{i=1}^{N} \frac{\sum_{t=1}^{T}\left(\hat{\varepsilon}_{i t-1} \Delta \hat{\varepsilon}_{i t}-\hat{\lambda}_{i}\right)}{\left(\sum_{t=1}^{T} \hat{\varepsilon}_{i t-1}^{2}\right)}
$$

En donde $\left(\hat{\varepsilon}_{i t}\right)$ es estimado de la ecuación (1), $\left(\lambda_{i}=\frac{1}{2}\left(\hat{\sigma}_{i}^{2}-\hat{s}_{i}^{2}\right)\right)$, para lo cual $\left(\hat{\sigma}_{i}^{2}\right)$ es la varianza de largo plazo de $\left(\hat{\varepsilon}_{i t}\right)$ y $\left(\hat{s}_{i}^{2}\right)$ es la varianza contemporánea. El segundo grupo de estadísticos se basan en la razón de varianza, definida de la matriz de varianzas y covarianzas de largo plazo. El método de estimación es FMOLS, y se escoge básicamente ya que corrige los problemas de endogeneidad y heterogeneidad presentes en las pruebas de cointegración basadas en los residuales del modelo y estimadas por OLS. Una ampliación de esta metodología puede encontrarse en Pedroni (2000, 2004).

\section{Datos y Resultados Empíricos}

En esta sección se presentan los datos y resultados de las pruebas de raíces unitarias y cointegración en panel, así como los coeficientes de la relación de largo plazo estimados para el modelo a estimar en la ecuación (1).

\subsection{Datos}

Los datos para este trabajo fueron obtenidos del Banco Mundial, a saber, se emplean datos sobre PIB (en millones de dólares de 2005), Formación bruta de capital fijo (en millones de dólares de 2005), Fuerza laboral (en millones de personas) y Patentes (número de registros). La base de datos 
abarca el periodo comprendido entre el año 1990 y el año 2010. En el Apéndice A se presentan las Gráficas de las 4 series para los 10 países.

\subsection{Prueba de Raíces Unitarias}

Según la Tabla 1, las 6 pruebas de raíces unitarias aplicadas a las 4 series de tiempo indican que en niveles estas series tienen raíz unitaria ya que la probabilidad de las pruebas no permite rechazar la hipótesis nula de la existencia de raíz unitaria, en el caso de la prueba de Hadri (2000), la hipótesis nula es que la serie es estacionaria, por lo cual como se ve se rechaza esta al 1\% de significancia. Por otra parte, la Tabla 2 presenta los resultados de las pruebas aplicadas a la primera diferencia de cada serie, y se evidencia que estás ya no tienen raíz unitaria, es decir, son estacionarias. En resumen, los resultados de las pruebas de raíces unitarias sobre las variables que son incluidas en el modelo, muestran que las 4 series son I(1), es decir, están integradas de orden uno.

Tabla 1. Resultados Pruebas de Raíces Unitarias (Niveles) - Primera Generación

\begin{tabular}{lcccccccc}
\hline \multirow{2}{*}{ Prue ba } & \multicolumn{2}{c}{ Ln(Y) } & \multicolumn{2}{c}{ Ln(L) } & \multicolumn{2}{c}{ Ln(K) } & \multicolumn{2}{c}{ Ln(PAT) } \\
\cline { 2 - 8 } & Estadístico & Prob. & Estadístico & Prob. & Estadístico & Prob. & Estadístico & Prob. \\
\hline Levin, Lin \& Chu t* & 0.098 & 0.5390 & -0.418 & 0.3381 & 1.069 & 0.8576 & 1.553 & 0.9398 \\
Breitung t-stat & -0.627 & 0.2654 & 0.516 & 0.6972 & -2.932 & 0.0017 & 1.016 & 0.8452 \\
Im, Pesaran and Shin W-stat & 0.065 & 0.5261 & 0.609 & 0.7289 & -2.537 & 0.0056 & 0.459 & 0.6768 \\
ADF - Fisher Chi-square & 17.106 & 0.6461 & 16.843 & 0.6632 & 37.184 & 0.0111 & 27.375 & 0.1250 \\
PP - Fisher Chi-square & 7.053 & 0.9965 & 15.928 & 0.7211 & 13.276 & 0.8653 & 29.739 & 0.0742 \\
Hadri Z-stat & 9.57173 & 0.0000 & 9.83644 & 0.0000 & 7.62121 & 0.0000 & 5.15155 & 0.0000 \\
\hline
\end{tabular}

Tabla 2. Resultados Pruebas de Raíces Unitarias (Diferencia) - Primera Generación

\begin{tabular}{lcccccccc}
\hline \multirow{2}{*}{ Prueba } & \multicolumn{2}{c}{$\Delta \mathbf{L n}(\mathbf{Y})$} & \multicolumn{2}{c}{$\Delta \mathbf{L n}(\mathbf{L})$} & \multicolumn{2}{c}{$\Delta \mathbf{L n}(\mathbf{K})$} & \multicolumn{2}{c}{$\Delta \mathbf{L n}(\mathbf{P A T})$} \\
\cline { 2 - 8 } & Estadístico & Prob. & Estadístico & Prob. & Estadístico & Prob. & Estadístico & Prob. \\
\hline Levin, Lin \& Chu t* & -7.305 & 0.0000 & -6.409 & 0.0000 & -7.686 & 0.0000 & -6.331 & 0.0000 \\
Breitung t-stat & -3.224 & 0.0006 & -4.157 & 0.0000 & -3.817 & 0.0001 & -1.711 & 0.0435 \\
Im, Pesaran and Shin W-stat & -4.853 & 0.0000 & -5.714 & 0.0000 & -4.764 & 0.0000 & -7.186 & 0.0000 \\
ADF - Fisher Chi-square & 56.310 & 0.0000 & 67.291 & 0.0000 & 55.752 & 0.0000 & 81.950 & 0.0000 \\
PP - Fisher Chi-square & 53.700 & 0.0001 & 71.627 & 0.0000 & 62.785 & 0.0000 & 96.448 & 0.0000 \\
Hadri Z-stat & 0.29898 & 0.3825 & 1.14229 & 0.1267 & -0.34925 & 0.6365 & 0.67438 & 0.2500 \\
\hline
\end{tabular}

La Tabla 3, presenta el resultado de la prueba de estacionariedad de Hadri y Rao (2008) aplicada a cada una de las series. Esta prueba, como se mencionó anteriormente incorpora en su prueba un quiebre estructural y la dependencia cross-section. En la columna "Quiebre" se presenta el año en el cual según la prueba existió un quiebre estructural para cada una de las series y cada uno de los países. En la columna 2 y 3 se muestra el modelo escogido para cada serie y país y el orden del proceso AR para el bootstraping. La prueba nos da evidencia para rechazar la hipótesis nula de estacionariedad para cada una de las series. ${ }^{3}$

\footnotetext{
${ }^{3}$ La prueba también se aplica para la serie en diferencias, reforzando los resultados de las pruebas de primera generación, es decir, las series son integradas de orden uno, estacionarias en primeras diferencias. Los resultados no se presentan aquí por espacio.
} 
Tabla 3. Resultado Prueba de Raíce Unitaria - Segunda Generación

\begin{tabular}{|c|c|c|c|c|c|c|c|c|c|c|c|c|}
\hline & \multicolumn{3}{|c|}{$\operatorname{Ln}(Y)$} & \multicolumn{3}{|c|}{$\operatorname{Ln}(K)$} & \multicolumn{3}{|c|}{$\operatorname{Ln}(L)$} & \multicolumn{3}{|c|}{$\operatorname{Ln}(\mathbf{P A T})$} \\
\hline & Quiebre & Model & $\mathbf{A R}$ & Quiebre & Model & $\mathbf{A R}$ & Quiebre & Model & $\mathbf{A R}$ & Quiebre & Model & $\mathbf{A R}$ \\
\hline ARG & 2001 & 3 & 1 & 2001 & 3 & 1 & 2004 & 3 & 4 & 2001 & 3 & 3 \\
\hline BRA & 2004 & 2 & 3 & 2002 & 3 & 0 & 1993 & 2 & 1 & 1997 & 1 & 4 \\
\hline CHI & 1997 & 2 & 1 & 1999 & 3 & 0 & 2001 & 3 & 4 & 2009 & 2 & 1 \\
\hline COL & 1999 & 3 & 2 & 1999 & 3 & 2 & 2004 & 3 & 2 & 2001 & 1 & 4 \\
\hline ECU & 1999 & 3 & 0 & 1999 & 3 & 1 & 2001 & 3 & 0 & 2001 & 1 & 0 \\
\hline MEX & 2009 & 1 & 1 & 1995 & 1 & 1 & 1999 & 3 & 4 & 1999 & 0 & 4 \\
\hline PAN & 2002 & 3 & 3 & 2001 & 1 & 4 & 1997 & 2 & 2 & 1996 & 1 & 0 \\
\hline PRY & 2000 & 3 & 0 & 1999 & 1 & 0 & 1997 & 1 & 1 & 2002 & 3 & 3 \\
\hline PER & 2001 & 3 & 3 & 2000 & 1 & 0 & 2003 & 1 & 0 & 2009 & 2 & 4 \\
\hline URY & 2002 & 3 & 0 & 2002 & 3 & 1 & 2003 & 1 & 0 & 1997 & 3 & 4 \\
\hline $\begin{array}{l}\text { Estadístico LM } \\
\text { P-Value Asintótico } \\
\text { P-Value Bootstrap }\end{array}$ & & $\begin{array}{l}.589181 \\
0.000 \\
0.046\end{array}$ & & & $\begin{array}{l}.325092 \\
.27 \mathrm{E}-10 \\
.026004\end{array}$ & & & $\begin{array}{l}15.83395 \\
0.0000 \\
0.0469\end{array}$ & & & $\begin{array}{c}22.390 \\
0.0000 \\
0.00514\end{array}$ & \\
\hline
\end{tabular}

Nota: El tipo de modelo y el orden del proceso AR, se seleccionaron por medio del criterio de información bayesiano.

\subsection{Prueba de Cointegración y Coeficientes de Largo Plazo}

A continuación se presentan los resultados de la prueba de Cointegración para el modelo panel. La Tabla 4 presenta los resultados de la Prueba de Cointegración de Pedroni, la cual como se presentó en la sección anterior está basada en los residuos de la regresión estimada por FMOLS.

Tabla 4. Resultado Prueba de Cointegración - Pedroni

\begin{tabular}{l|cc}
\hline & Estadístico & Prob. \\
\hline Panel v-Statis tic & 7.892833 & 0.0000 \\
Panel rho-Statis tic & 0.511618 & 0.6955 \\
Panel PP-Statistic & -3.004954 & 0.0013 \\
Panel ADF-Statis tic & -1.00323 & 0.1579 \\
Group rho-Statis tic & 1.729542 & 0.9581 \\
Group PP-Statis tic & -3.684522 & 0.0001 \\
Group ADF-Statistic & -1.586752 & 0.0791 \\
\hline
\end{tabular}

Los resultados sugieren, por mayoría, que se rechaza la hipótesis nula de No Cointegración al $10 \%$ de significancia, a favor de la existencia de una relación de cointregación entre las variables. En la Tabla 5 se muestran los coeficientes estimados de la relación de largo plazo de la ecuación (1) a nivel individual y en panel. 
Tabla 5. Coeficientes de la relación de largo plazo, individuales y panel.

\begin{tabular}{cccl}
\hline & $\boldsymbol{L} \boldsymbol{n}(\boldsymbol{K})$ & $\boldsymbol{L} \boldsymbol{n}(\boldsymbol{L})$ & $\boldsymbol{L n}(\boldsymbol{P} \boldsymbol{A} \boldsymbol{T})$ \\
\hline ARG & $0.376928^{* * *}$ & $0.71871^{* * *}$ & $0.018203^{*}$ \\
BRA & $0.34267^{* * *}$ & $0.916409^{* * *}$ & $-0.01773^{*}$ \\
CHI & $0.145148^{* * *}$ & $1.412513^{* * *}$ & $0.15478^{*}$ \\
COL & $0.285603^{* * *}$ & $0.484159^{* * *}$ & $0.01534^{*}$ \\
ECU & $0.468429^{* * *}$ & $0.326083^{* * *}$ & $0.040717^{*}$ \\
MEX & $0.237426^{* * *}$ & $0.682447^{* * *}$ & $0.034054^{*}$ \\
PAN & $0.182271^{* * *}$ & $0.876806^{* * *}$ & 0.144426 \\
PRY & $0.304278^{* * *}$ & $0.630998^{* * *}$ & $0.06652^{* * *}$ \\
PER & $0.190432^{* * *}$ & $0.870465^{* * *}$ & $0.04022^{* *}$ \\
URY & $0.24603^{* * *}$ & $1.798304^{* * *}$ & $0.04083^{*}$ \\
\hline PANEL & $0.335275^{* * *}$ & $0.60474^{* * *}$ & $0.055063^{* * *}$ \\
\hline
\end{tabular}

Podemos interpretar los resultados anteriores de la siguiente manera, por ejemplo, para los coeficientes del Panel, ante un incremento del 1\% en el Capital, a largo plazo, la producción se incrementa en $0.33 \%$. Un incremento del $1 \%$ en la fuerza laboral, en el largo plazo, incrementa el PIB en un $0.60 \%$. Finalmente, y parte del propósito de este estudio empírico, ante un incremento de las Patentes en un 1\%, a largo plazo, provoca un incremento del PIB. A nivel individual es curioso el resultado de Brasil, ya que un coeficiente asociado a las Patentes negativo no tiene mucho sentido económico en la vía del supuesto de que el registro de patentes tiene un efecto positivo sobre el PIB. Panamá y Chile tienen los coeficientes asociados al número de patentes más altos de la región. Por otra parte Colombia y Argentina presentan el coeficiente más bajo de los 10 países que hacen parte de la muestra.

\section{Conclusiones}

En este documento se estudió empíricamente la relación entre Patentes y crecimiento económico para 10 países de América Latina, utilizando información anual sobre el número de patentes y PIB, durante el periodo 1990 - 2010. Adicionalmente, se controla esta relación con otros factores de producción como Capital y Trabajo.

Se aplican pruebas de raíces unitarias de primera generación, como la IPS (2003), LLC (2002), Breitung (2000), Hadri (2000), y de segunda generación, como la prueba propuesta por Hadri y Rao (2008), con el fin de determinar el orden de integración de las series. Estas pruebas determinan que las series del modelo son integradas de orden uno.

Los resultados presentan evidencia estadística sobre la existencia de una relación de largo plazo entre las Patentes, el Capital, el Trabajo y el PIB para los 10 países considerados. Ante un incremento del $1 \%$ en el capital, a largo plazo, el PIB se incrementa en $0.33 \%$ aproximadamente. Un incremento del $1 \%$ en la fuerza laboral, en el largo plazo, incrementa el PIB en un $0.60 \%$. 
Finalmente, y parte del propósito de este estudio empírico, ante un incremento de las Patentes en un $1 \%$, a largo plazo, provoca un incremento del PIB.

Se presenta entonces un resultado interesante, y es el impacto que tienen las Patentes sobre el PIB y por ende sobre el crecimiento económico de los países. Una posible explicación a la baja elasticidad del PIB con respecto a las patentes es que en la mayoría de países de América Latina, la mayoría de los registros de patentes son efectuados por no residentes, siendo el registro de los residentes muy pequeño en términos relativos. Lo anterior también se debe a la poca innovación que se presenta en los países latinos.

Siendo estrictos con los resultados aquí presentados, se debe en futuras investigación tratar de ampliar el número de observaciones de tiempo, ya que esta puede ser una limitación en los resultados. Adicionalmente, en este documento se estudia una hipótesis que se resume en, a mayor nivel de actividad innovadora (Patentes), mayor es la tasa de crecimiento económico; se debe entonces también probar la calidad en la actividad innovadora, no solo la cantidad (Hasan y Tucci (2010)). Y finalmente, un punto de suma importancia para las políticas al sistema de propiedad industrial es evaluar si es mayor el efecto de las patentes registradas por residentes o las registradas por no residentes. 


\section{Referencias}

Barro, R. (1991). "Economic Growth in a Cross Section of Countries". The Quarterly Journal of Economics, MIT Press, Vol. 106(2), pp. 407 - 443.

Breitung, J. (2000). "The local power of some unit root tests for panel data". Advances in Econometrics, Vol. 15, pp. 161 - 177.

Campo, J., Cantor, N., Herrera, J.P., De Quinto, M. y Sánchez, D. (2012). "Costrucción de un índice de regionalización para el Sistema Nacional de Propiedad Industrial (SPI): Una aproximación desde la metodología de componentes principales”. Documentos de Trabajo, No. 1, Superintendencia de Industria y Comercio.

Chang, Y. (2004). "Bootstrap unit root test in panel data". Journal of Econometrics, Vol. 120, pp. 263 - 293.

Chen, Y. y Puttitanun, T. (2005). "Intellectual property rights and innovation in developing countries", Journal of Development Economics, vol. 78, pp. 474 - 493.

Choi, I. (2001). "Unit root test for panel data". Journal of International Money and Finance, vol 20, pp. $249-272$.

Cysne, R. y Turchick, D. (2012). "Intellectual property rights protection and endogenous economic growth revisited", Journal of Economic Dynamics and Control, vol. 36, pp. 851 - 861.

Engle, R.F. y Granger, C. (1987). Co-integration y Error- Correction: Representation, estimation y testing. Econometrica, No. 55 (2), pp. $251-276$.

Dichey, D. y Fuller, W. (1979). "Distribution of the Estimators for Autoregressive Time Series with a Unit Root", Journal of the American Statistical Association, Vol. 74, pag. 427 - 431.

Breitung, J. (2000). "The local power of some unit root tests for panel data". Advances in Econometrics, vol 15, pp. $161-177$.

Entorf, H. (1997). "Random walks with drifts: Nonsense regression and spurious fixed-effect estimation", Journal of Econometrics, Vol. 80, pp. 287 - 296.

Fink, C. y Maskus, K. (2005). "Intellectual Property and Development”, World Bank, Washington, DC.

Futagami, K. y Iwaisako, T. (2007). "Dynamic analysis of patent policy in a endogenous gr4owth model”, Journal of Economic Theory, vol. 132, pp. 306 - 334.

Granger, C. y Newbold, P. (1974). "Spurious Regressions in Econometrics", Journal of Econoemtrics, Vol. 2, pag. 111 - 120.

Gilbert, R. y Shapiro, C. (1990). "Optimal patent length and breadth", Journal of Economics, vol. 21, pp. $106-112$.

Gould, D. y Gruben, W. (1996). "The role of intelectual property rights in economic growth", Journal of Development Economics, vol. 48, pp. 323 - 350.

Hadri, K. (2000). "Testing for stationarity in heterogeneous panel data". Econometric Journal, vol 3, pp. $148-161$.

Hadri, K. y Rao, Y. (2008). "Panel Stationarity Test with Structural Breaks". Oxford Bulletin of Economics and Statistics, Vol. 70, No. 2, pp. 245 - 269.

Hasan, I. y Tucci, C. (2010). “The innovation-economic growth nexus: Global evidence”, Research Policy, vol. 39, pp. $1264-1276$. 
Hu, A. y Png, I. (2012). "Patent Rights and Economic Growth: Evidence from Cross-Country Panels of Manufacturing Industries", Deparment of Economics, National University of Singapore.

Im, K., Pesaran, M. y Shin, Y. (2003). "Testing for unit roots in heterogeneous panels". Journal of Econometrics, vol 115, pp. $53-74$.

Judd, K. (1985). “On the performance of patents”, Econometrica, vol. 53, pp. 567 - 585.

Kao, C. (1999). "Spurious regression and residual-based test for cointegration in panel data", Journal of Econometrics, Vol. 90, pp. 1 - 44.

Kim, Y. (1997). "Imitation to Innovation: The Dynamics of Korea`s Technological Learning", Harvard Business School Press, Boston.

Kim, Y., Lee, K., Park, W. y Choo, K. (2012). “Appropriate intelectual property protection and economic growth in countries at different levels of development", Research Policy, vol. 41, pp. $358-375$.

Koléda, G. (2004). "Patents novelty requirement and endogenous growth", Revue d'économie politique, vol. 114, pp. $201-221$.

Kwiatkowski, D., Phillips, P., Schmidt, P. y Shin, Y. (1992). "Testing the null Hypothesis of Stationarity Against the Alternative of Unit Root", Journal of Econometrics, Vol 54 (1 - 3), pag. $159-178$.

Levin, A., Lin, C. and Chu, C. (2002). "Unit Root Test in Panel Data: Asymptotic and Finitesample Properties". Journal of Econometrics, Vol. 108, pp. 1 - 24.

Maddala, G. y Wu, S. (1999). "A comparative study of unit root test with panel data y a new simple test". Oxford Bulletin of Economics y Statistics, vol 61, pp. 631 - 652.

Park, W. y Ginarte, J. (1997). "Intellectual property rights and economic growth", Contemporany Economic Policy, vol. 15, pp. 51 - 61.

Park, W. (2008). “International patent protection: 1960 - 2005”, Research Policy, vol. 37, pp. 761 766.

Pedroni, P. (1999). "Critical Values for Cointegration Test in Heterogeneous Panels with Multiple Regressors”. Oxford Bulletin of Economics y Statistics, Special Issue 0305-9049.

Pedroni, P. (2000). "Fully modified OLS for heterogeneous cointegrated panels". Advances in Econometrics, Vol 15, pp. $93-130$.

Pedroni, P. (2004). "Panel Cointegration: asymptotic y finite sample propierties of pooled time series with an application to the PPP hypothesis: New Results". Econometric Theory, vol 20, pp. $597-627$.

Tandon, P. (1982). "Optimal patents with compulsory licensing", Journal of Political Economy, vol. 90 , pp. $470-489$.

Schneider (2005). "International trade, economic growth and intellectual property rights: A panel data study of developed and developing countries", Journal of Development Economics, vol. 78, pp. $529-547$. 
Apéndice A. Gráfico de las Series por País

Argentina

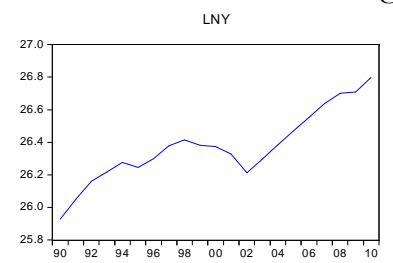

LNK

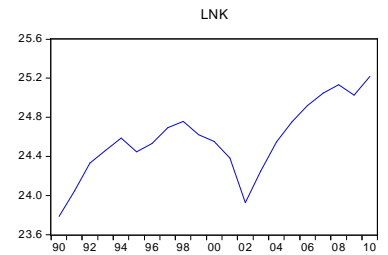

Chile

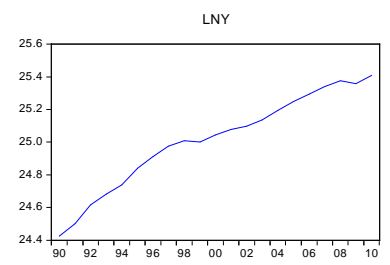

LNK

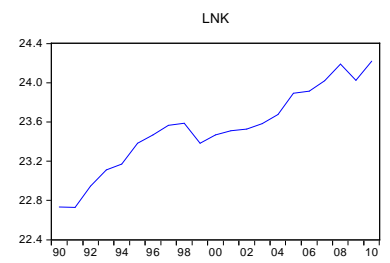

Ecuador

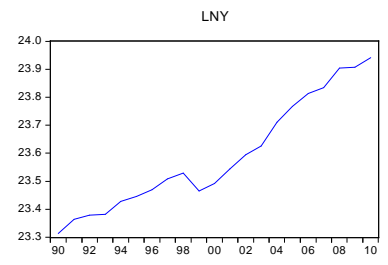

LNK

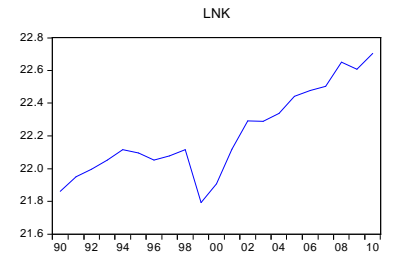





LNK

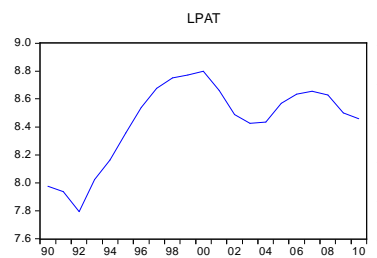

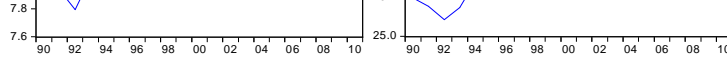

Brasil
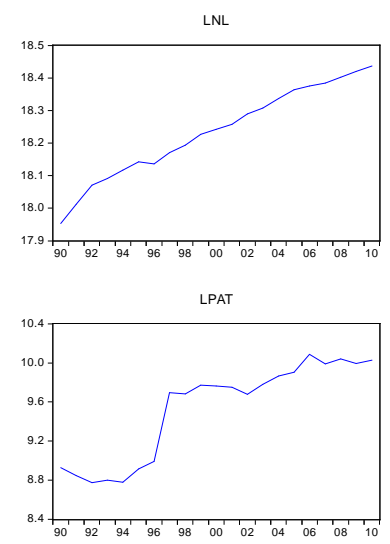

Colombia

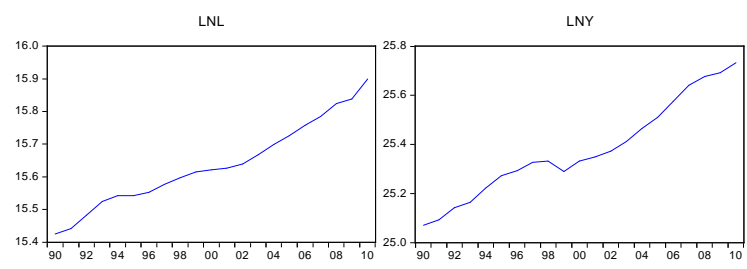

LNK
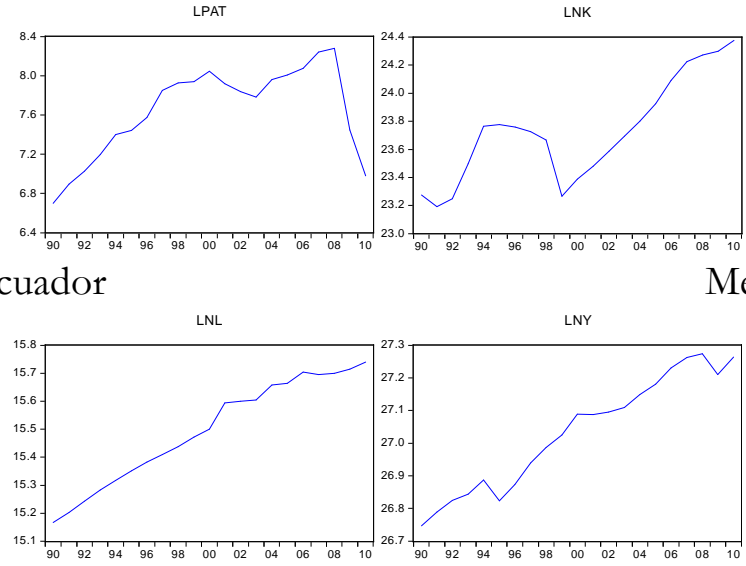

LNK

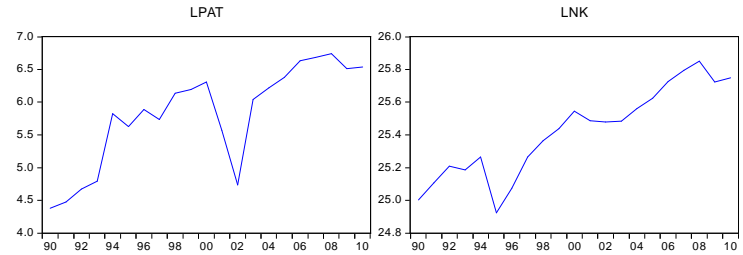

LNL



LNPAT

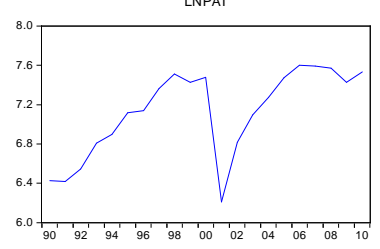

México

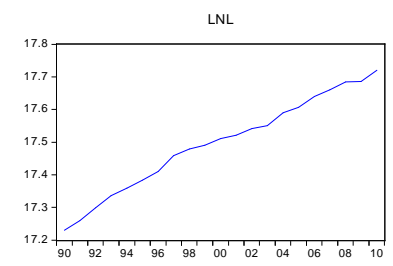

LPAT

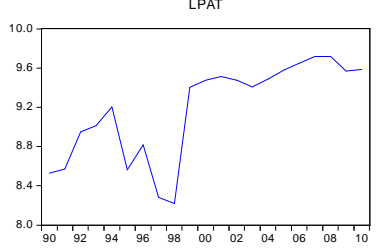



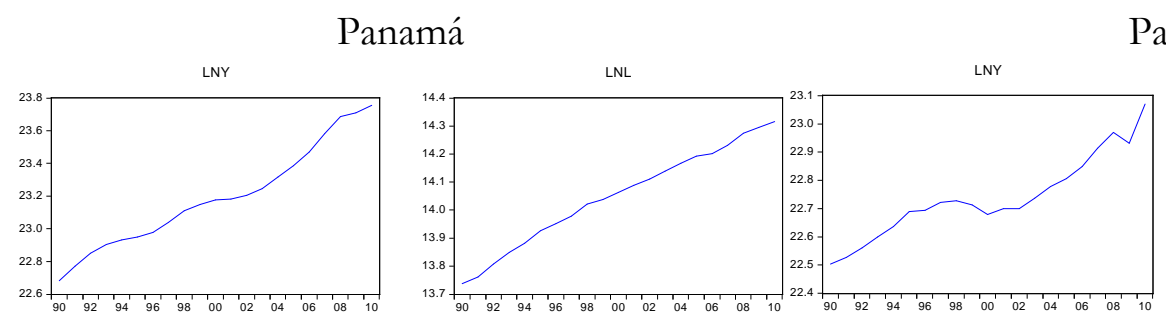

Paraguay

LNK
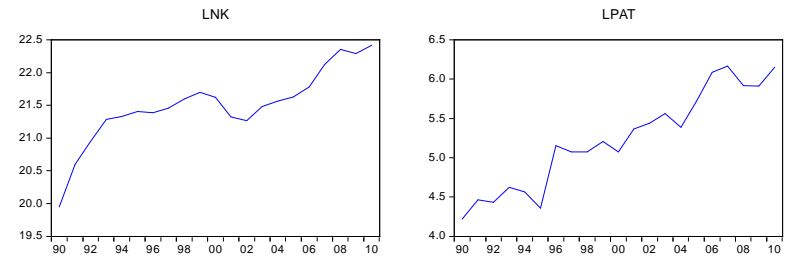

Perú

LNY

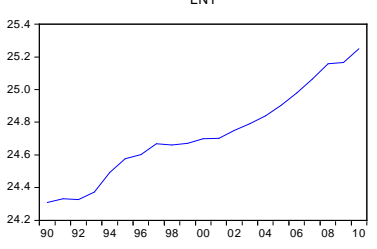

LNK
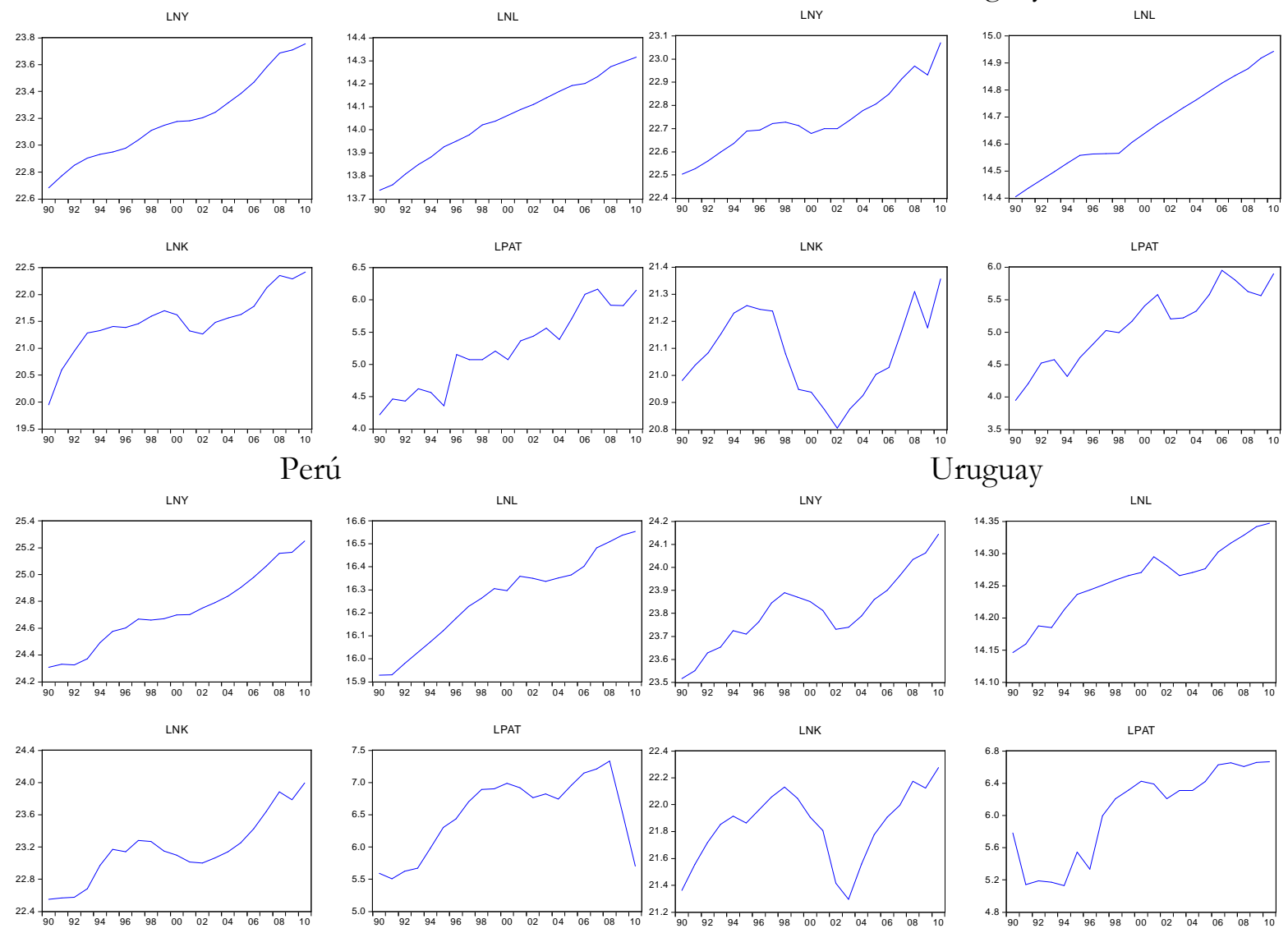

Uruguay

LNY
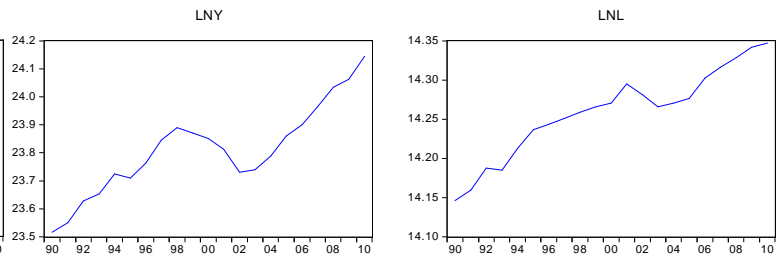

LPAT

LNK
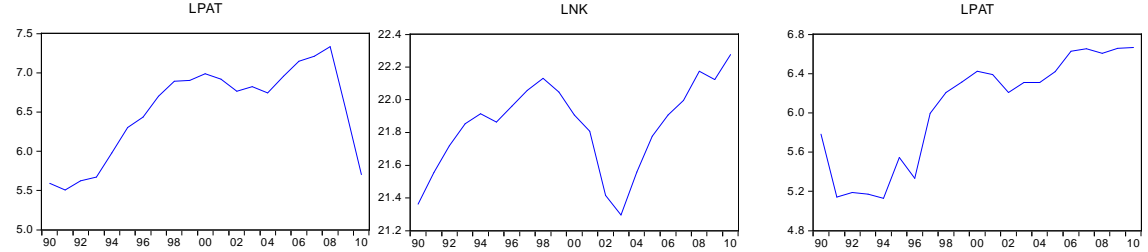